\title{
Angina in granulomatosis with polyangiitis: A case report
}

\author{
HUIJIE DONG $^{1 *}$, DAYUAN LOU ${ }^{1 *}$, XIAOFEI JI ${ }^{2}$ and PENG QU ${ }^{1}$ \\ ${ }^{1}$ Department of Cardiology, The Second Affiliated Hospital of Dalian Medical University, Dalian, Liaoning 116023; \\ ${ }^{2}$ Department of Neurology, The First Affiliated Hospital of Dalian Medical University, Dalian, Liaoning 116011, P.R. China
}

Received June 21, 2017; Accepted August 7, 2017

DOI: $10.3892 /$ etm.2017.4946

\begin{abstract}
Granulomatosis with polyangiitis (GPA) often causes small blood vessel lesions in multiple organs, with less impact on large vessels. We report a case involving not only large blood vessels, but also angina. The computed tomographic angiography of large vessels and vascular ultrasonography of the extremity also suggested great arteries of the body were involved. Further cytoplasmic antineutrophil cytoplasmic antibody tests, pathological examination and dynamic chest computed tomography scan confirmed the diagnosis of GPA. Anti-inflammatory therapy and coronary secondary prevention were given after diagnosis by pathological examination and laboratory evaluation. The symptoms of angina pectoris were relieved. This rare case report reminds us that among the well-known risk factors of atherosclerotic diseases, GPA is also an important risk factor for acute coronary syndrome. Early foresight for this rare risk factor can improve the early diagnosis and treatment of the disease, which will improve the prognosis of the patients.
\end{abstract}

\section{Introduction}

Granulomatosis with polyangiitis (GPA), formerly known as Wegener's granulomatosis, is a granulomatous disorder that most commonly presents with involvement of the sinuses, lungs, and kidneys. Cardiac involvement is uncommon, and is reported in $\sim 8-44 \%$ of cases with Wegener's granulomatosis (1), which commonly manifested as pericarditis, myocarditis and arrhythmias. It can eventually lead to high

Correspondence to: Dr Xiaofei Ji, Department of Neurology, The First Affiliated Hospital of Dalian Medical University, 222 Zhongshan Road, Xigang, Dalian, Liaoning 116011, P.R. China E-mail: jixiaofei1979@163.com

Dr Peng Qu, Department of Cardiology, The Second Affiliated Hospital of Dalian Medical University, 467 Zhongshan Road, Shahekou, Dalian, Liaoning 116023, P.R. China

E-mail: qqn8dd@163.com

*Contributed equally

Key words: angina, atherosclerosis, granulomatosis with polyangiitis, acute coronary syndrome, rheumatic immunity blood pressure and heart failure. In addition, because GPA often causes small blood vessel lesions, there are only a small number of clinical reports of acute coronary syndrome caused by this disease (2-5).

In the clinical diagnosis of coronary heart disease, hypertension, diabetes and smoking are the most common risk factors for atherosclerosis. Effective treatments such as blood pressure control, hypoglycemic management and smoking cessation have been shown effectively to reduce the recurrence of cardiovascular disease. However, there are still $\sim 15 \%$ coronary heart disease patients with unclear mechanism of atherosclerosis. This includes the large arterial inflammatory change caused by GPA. There is often not enough emphasis on this risk factor to delay the patient's diagnosis and treatment in clinical work.

In this study, we report a rare case that suffered angina involved in aortic atherosclerosis with GPA.

\section{Case report}

We report a rare case of a 64-year-old man who was admitted to the hospital in April, 2015 with a 2-week history of chest discomfort, fever, headache, dizziness and bloody, purulent rhinorrhea. He denied a family history of cardiac disease and did not smoke. This study was approved by the Ethics Committee of the First Affiliated Hospital of Dalian Medical University. Signed written informed consents were obtained from the case. He had hypertension for 40 years, controlled with $150 \mathrm{mg}$ irbesartan, $30 \mathrm{mg}$ nifedipine, and $25 \mathrm{mg}$ metoprolol. He also had diabetes mellitus for 6 years, and was taking glibenclamide $2 \mathrm{mg}$ /day and acarbose $150 \mathrm{mg}$ /day. He had a cerebral hemorrhage 15 years prior. Based on a history of dizziness and brain computed tomography (CT), he was diagnosed multiple times with acute cerebral infarction in the previous 13 years. Because of poor blood pressure control, he underwent a comprehensive physical examination one year previously. He was found to have diffuse atherosclerotic plaques involving the aorta, as well as carotid, iliac, renal and limb arteries. The right internal carotid artery, right external iliac artery, and left superficial femoral artery were occluded. The left renal artery had severe stenosis (Fig. 1). After changing the antihypertensive drugs, the blood pressure remained well controlled. He had no discomfort, and took aspirin and atorvastatin as usual.

On admission, he complained of chest discomfort and fever. Blood pressure was $113 / 93 \mathrm{mmHg}$, heart rate was 

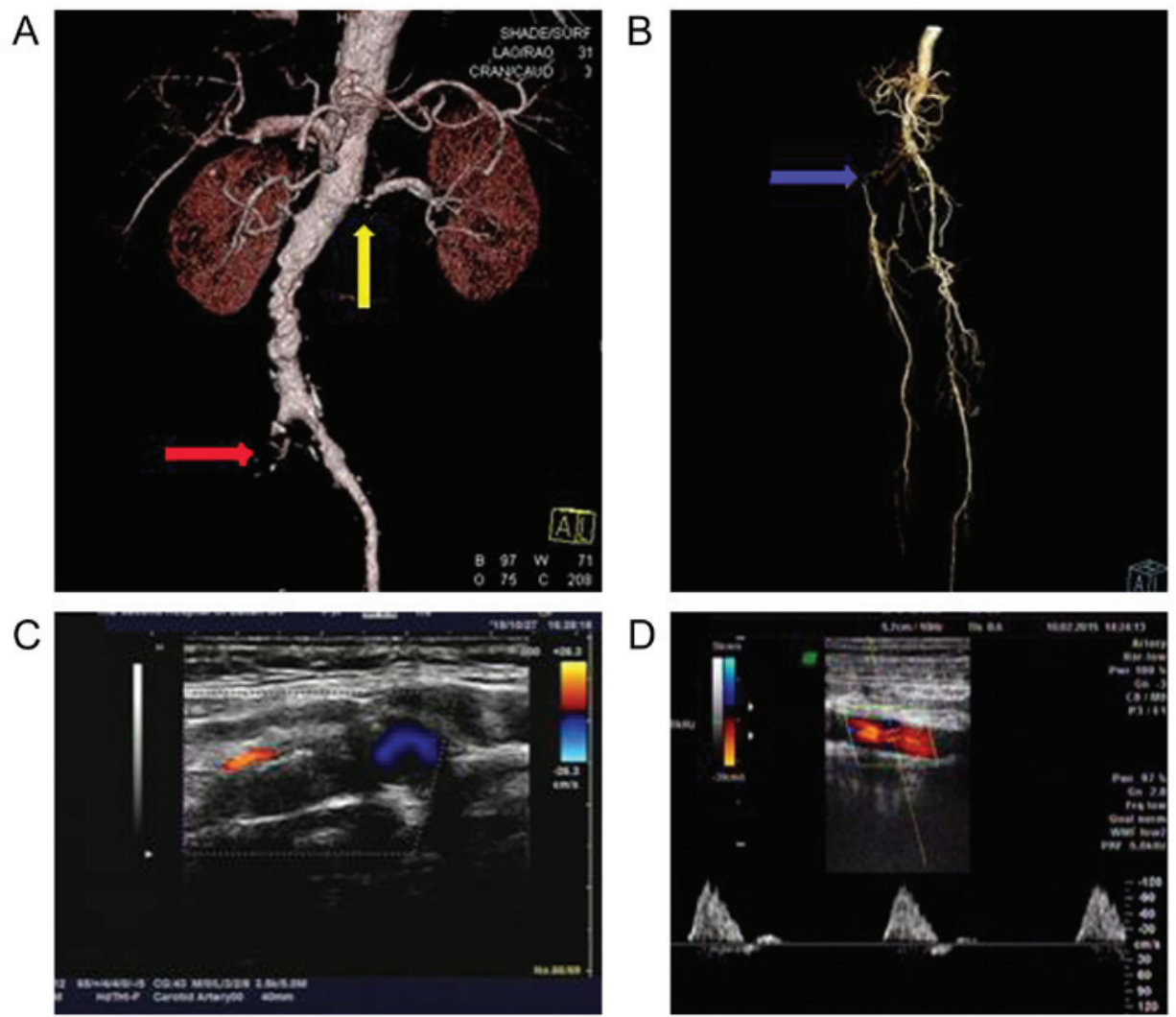

Figure 1. Comprehensive physical examination in 2015. (A and B) CT angiography of the renal (yellow arrow), iliac (red arrow) and femoral arteries (blue arrow). (C) Carotid ultrasound. (D) Axillary artery ultrasound.

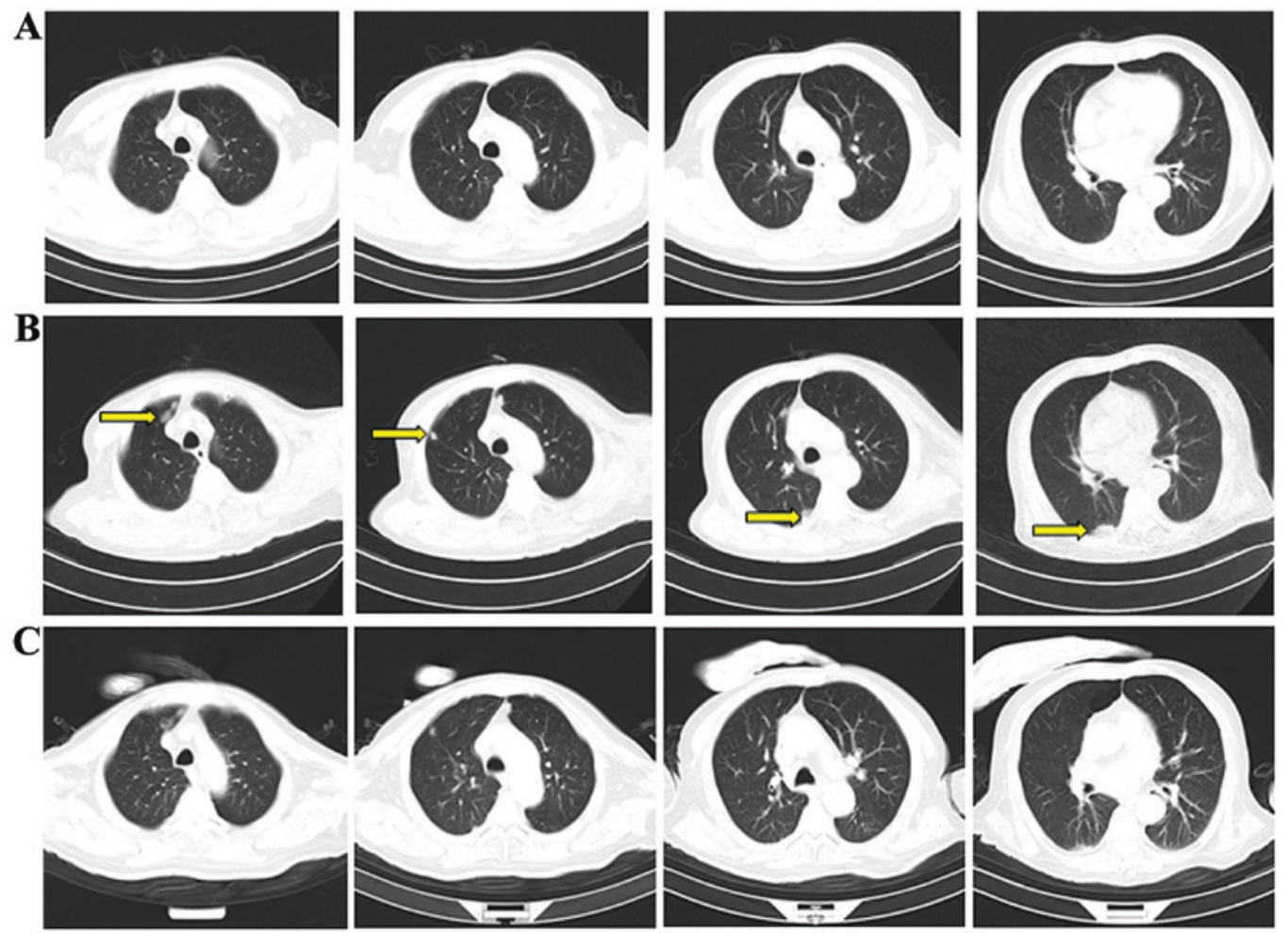

Figure 2. The dynamic changes of the lung CT scan. (A) The lung CT in 2015. (B) The lung CT in 2016 without hormone therapy. Arrows indicate granulomatous nodules. (C) The lung CT in 2016 after hormone therapy.

64 beats/min; there were no respiratory or cardiac pathological findings on auscultation. We performed blood tests and CT scans (Fig. 2). There were significant changes in the same parameters tested one year prior (Table I). 
Table I. Blood biochemistry inspection.

\begin{tabular}{lccccc}
\hline Date & WBC $\left(\mathrm{x} 10^{9} / \mathrm{l}\right)$ & HGB $(\mathrm{g} / \mathrm{l})$ & $\mathrm{Cr}(\mu \mathrm{mol} / \mathrm{l})$ & $\mathrm{CRP}(\mu \mathrm{g} / \mathrm{ml})$ & $\mathrm{ESR}(\mathrm{mm} / \mathrm{h})$ \\
\hline 2015.02 & 9.58 & 157 & 117 & 12 & 2 \\
2016.07 & 10.65 & 140 & 148 & 148 & 35 \\
\hline Date & C-ANCA & P-ANCA & Anti-PR3 & IgG $(\mathrm{g} / \mathrm{l})$ & $\operatorname{IgA}(\mathrm{g} / \mathrm{l})$ \\
\hline 2015.02 & - & + & + & 13.5 & 2.15 \\
2016.07 & + & - & + & 23.7 & 4.04 \\
\hline Date & $\mathrm{C} 3(\mathrm{~g} / \mathrm{l})$ & Urine RBC/HP & Proteinuria $(\mathrm{mg} / 24 \mathrm{~h})$ & $\mathrm{EF}(\%)$ & $\mathrm{cTNI}(\mu \mathrm{g} / \mathrm{l})$ \\
\hline 2015.02 & 1.1 & 0.15 & 357.42 & 56 & 0.06 \\
2016.07 & 0.8 & 42.6 & 1147.5 & 58 & 0.01 \\
\hline
\end{tabular}

HGB, hemoglobin; Cr, creatinine; CRP, C-reactive protein; ESR, erythrocyte sedimentation rate; EF, ejection fraction.

The patient underwent otolaryngologic evaluation for a bloody, purulent, bilateral nasal discharge. A biopsy of a right nasal sepal tumor showed a squamous epithelium with fibrous hyperplasia, many lymphocytes, plasma cells, neutrophil infiltration and leukemia-like vasculitis with local epithelial erosion. Based on the symptoms and examination results, rheumatology diagnosed granulomatous vasculitis. Because the patient had renal insufficiency and pneumonia, the family did not agree to coronary angiography. He then received glucocorticoids, cyclophosphamide, anti-inflammatory agents and coronary secondary prevention therapy. The fever and chest pain improved significantly. Pulmonary nodules also diminished.

\section{Discussion}

The annual incidence of granulomatous vasculitis is $\sim 0.4 / 100,000$. It can present at any age, but is more common between the ages of 30-50 years. The male:female ratio is 1.6:1. The etiology has not been fully elucidated. It is easily misdiagnosed because the lesions are often limited to the upper respiratory tract in the early stage of disease. Approximately $6-44 \%$ of granulomatous vasculitis patients have comorbid pericarditis, myocarditis and coronary arteritis with cardiac involvement; of these, $50 \%$ have coronary artery vasculitis. In patients with atherosclerosis, GPA can accelerate the process, with a significant increase in morbidity and mortality due to cardiovascular disease, and a poor prognosis (6-8).

This patient had fever, upper respiratory tract symptoms, blood in the urine, multiple bilateral lung nodules, and renal lesions, and was positive for cytoplasmic antineutrophil cytoplasmic antibodies (C-ANCA). Histopathological examination of a sinus neoplasm indicated necrotizing vasculitis. The findings conformed to the 1990 American Rheumatism Association criteria for the diagnosis of granulomatous vasculitis. In addition, the patient's chest pain and electrocardiogram were diagnostic of angina pectoris. This middle-aged male patient had hypertension, diabetes, and other risk factors for atherosclerosis, and was found to have diffuse arterial plaques, partial occlusion and severe stenosis. These factors did not completely explain the coronary arterial lesions of GPA. However, the patient had incipient angina accompanied by GPA. This suggested the development of angina pectoris was associated with a rheumatologic disorder.

The patient had a history of hypertension for 40 years, and sustained kidney damage, recurrent cerebral infarctions, and vascular sclerosis and occlusion in recent years. However, no rheumatologic history was apparent; therefore, we could not conclude that the vascular lesions and true cause of high blood pressure were associated with the presence of vasculitis. Histopathological characteristics of coronary artery anatomy may ultimately provide answers.

At present, the main treatment of granulomatous vasculitis is glucocorticoid alone or in combination with an immune inhibitor. This treatment is highly effective for granulomatous vasculitis with damage to the lung, brain, and heart. However, the treatment of granulomatous vasculitis with angina pectoris, or myocardial infarction with coronary artery lesions, remains controversial (9-11). Because there is no strong evidence for the effectiveness of percutaneous coronary intervention in coronary artery stenosis caused by GPA, we chose to use glucocorticoids and immune inhibitors, combined with conservative treatment for coronary prevention, and achieved good early results.

Although granulomatous vasculitis is rare, it is also a risk factor for cardiovascular and cerebrovascular disease. Therefore, even if there are clear risk factors for atherosclerosis in patients with acute coronary syndrome, the presence of unexplained fever should prompt consideration of GPA. This will be of great significance for treatment and prognosis.

\section{References}

1. Hoffman GS, Kerr GS, Leavitt RY, Hallahan CW, Lebovics RS, Travis WD, Rottem M and Fauci AS: Wegener granulomatosis: An analysis of 158 patients. Ann Intern Med 116: 488-498, 1992.

2. Schiefermueller J, Alaour B, Calver A and Curzen N: Lesson of the month 1: Beware the atypical presentation: eosinophilic granulomatosis with polyangiitis presenting as acute coronary syndrome. Clin Med (Lond) 17: 180-182, 2017. 
3. Benallegue N, Lozach P, Belizna C, Lavigne C and Urbanski G: Acute coronary vasospasm in a patient with eosinophilic granulomatosis with polyangiitis following NSAID administration: A case report. Medicine (Baltimore) 95: e5259, 2016.

4. Sokołowska B, Szczeklik W, Celejewska-Wójcik N, Macioł K, Stodółkiewicz E, Kaczmarek P, Nowakowski T, Mastalerz L and Musiał J: Exacerbation of eosinophilic granulomatosis with polyangiitis (EGPA) with heart involvement mimicking acute coronary syndrome. Przegl Lek 70: 991-992, 2013.

5. Asdonk T, Tiyerili V, Dörner J, Thomas D, Schaefer C, Skowasch D, Nickenig G and Pabst S: Acute coronary syndrom as a cardiac manifestation of granulomatosis with polyangiitis. Dtsch Med Wochenschr 138: 213-217, 2013 (In German).

6. Holle JU and Gross WL: Treatment of ANCA-associated vasculitides (AAV). Autoimmun Rev 12: 483-486, 2013.
7. Imbalzano E, Lo Gullo A, Costantino R, Tomasello C, Giugno V and Saitta A: Coronary vasculitis in granulomatosis with polyangiitis. Int J Cardiol 173: 593-595, 2014.

8. Jeong HC, Kim KH, Cho JY, Song JE, Yoon HJ, Seon HJ, Ahn Y, Jeong MH, Cho JG and Park JC: Cardiac involvement of churgstrauss syndrome as a reversible cause of dilated cardiomyopathy. J Cardiovasc Ultrasound 23: 40-43, 2015.

9. Knockaert DC: Cardiac involvement in systemic inflammatory diseases. Eur Heart J 28: 1797-1804, 2007.

10. Korantzopoulos P, Papaioannides D and Siogas K: The heart in Wegener's granulomatosis. Cardiology 102: 7-10, 2004.

11. Sulaiman W, Seung OP and Noor SM: Acute myocardial infarction as Eosinophilic granulomatosis with polyangiitis (formerly Churg Strauss syndrome) initial presentation. Rev Bras Reumatol 54: 393-396, 2014 (In Portuguese). 